Article

\title{
Functional and Genetic Diversity of Bacteria Associated with the Surfaces of Agronomic Plants
}

\section{Basharat Ali}

Department of Microbiology and Molecular Genetics, University of the Punjab, Lahore 54590, Pakistan; basharat.ali.mmg@pu.edu.pk; Tel.: +92-300-4297937

Received: 9 February 2019; Accepted: 2 April 2019; Published: 4 April 2019

Abstract: The main objective of this study was to evaluate the genetic diversity and agricultural significance of bacterial communities associated with the surfaces of selected agronomic plants (carrot, cabbage and turnip). The bacterial diversity of fresh agricultural produce was targeted to identify beneficial plant microflora or opportunistic human pathogens that may be associated with the surfaces of plants. Bacterial strains were screened in vitro for auxin production, biofilm formation and antibiotic resistance. 16S rRNA gene sequencing confirmed the presence of several bacterial genera including Citrobacter, Pseudomonas, Pantoea, Bacillus, Kluyvera, Lysinibacillus, Acinetobacter, Enterobacter, Serratia, Staphylococcus, Burkholderia, Exiguobacterium, Stenotrophomonas, Arthrobacter and Klebsiella. To address the biosafety issue, the antibiotic susceptibility pattern of strains was determined against different antibiotics. The majority of the strains were resistant to amoxicillin $(25 \mu \mathrm{g})$ and nalidixic acid $(30 \mu \mathrm{g})$. Strains were also screened for plant growth-promoting attributes to evaluate their positive interaction with colonized plants. Maximum auxin production was observed with Stenotrophomonas maltophilia MCt-1 (101 $\left.\mu \mathrm{g} \mathrm{mL}{ }^{-1}\right)$ and Bacillus cereus PCt-1 $\left(97 \mu \mathrm{g} \mathrm{mL}^{-1}\right)$. Arthrobacter nicotianae Lb-41 and Exiguobacterium mexicanum $\mathrm{MCb}-4$ were strong biofilm producers. In conclusion, surfaces of raw vegetables were inhabited by different bacterial genera. Potential human pathogens such as Bacillus cereus, Bacillus anthracis, Enterobacter cloacae, Enterobacter amnigenus and Klebsiella pneumoniae were also isolated, which makes the biosafety of these vegetable a great concern for the local community. Nevertheless, these microbes also harbor beneficial plant growth-promoting traits that indicated their positive interaction with their host plants. In particular, bacterial auxin production may facilitate the growth of agronomic plants under natural conditions. Moreover, biofilm formation may help bacteria to colonize plant surfaces to show positive interactions with host plants.

Keywords: food biosafety; bacterial colonization; antibiotic resistance; biofilm formation; raw-eaten vegetables; bacterial auxin production

\section{Introduction}

Fresh vegetables and fruits are integral components of the human diet and consumed in sufficient amounts to maintain good health [1]. There has been a correlation between foodborne outbreaks and increased production, imports and the consumption of fresh agricultural produce [2]. Raw vegetables can harbor pathogenic microorganisms that can cause serious illnesses to humans after consumption. Routes of microbial contamination include the application of organic wastes to soil as fertilizers, use of fecal-contaminated water for irrigation, direct contact with livestock and post-harvest hygiene issues [3]. In most common practices, fresh fruits and vegetables are consumed raw or processed to minimize the chances of foodborne outbreaks. The frequency of foodborne outbreaks from the consumption of contaminated vegetables and fruits has increased in recent decades. There is a long list of reports that confirms that raw vegetables foster several potentially pathogenic organisms, indicating their key involvement in fresh produce-associated outbreaks [4-9]. 
True or opportunistic human pathogens have been found and reported with foodborne outbreaks due to their adaptation to soil and plant surfaces [10]. They may be highly competitive for nutrients and produce antimicrobial agents to suppress the native microflora to colonize plant surfaces. For instance, pathogenic strains of Pseudomonas aeruginosa and Stenotrophomonas maltophila are well documented to colonize wheat and strawberries, respectively [11]. A similar ability of Burkholderia cepacia to cause infections in plants and humans has also been reported [12]. Besides the true human pathogens (Escherichia coli and Salmonella enterica), a number of potential human pathogens such as Achromobacter xylosoxidans, Janthinobacterium lividum, Alcaligenes xylosoxidans, Alcaligenes faecalis, Serratia marcescens, Staphylococcus aureus, Enterobacter amnigenus, Bacillus cereus, and Stenotrophomonas maltophilia have been reported to be present in plant-associated environments [13,14].

Plant-associated microorganisms can conveniently be categorized in three groups: plant-beneficial, plant pathogenic and opportunistic human pathogenic organisms. The plant-beneficial group of bacteria comprises members of different genera such as Burkholderia, Enterobacter, Stenotrophomonas, Pseudomonas, Herbaspirillum, Ralstonia, Bradyrhizobium, Ochrobactrum, Bacillus, Rhizobium and Staphylococcus. These microorganisms are mostly root-associated and factor in the bivalent interactions with plants and human or animal hosts [15]. The microorganisms of these genera proved themselves beneficial for plants by playing their role in growth promotion, protection from phytopathogens and provision of different elemental nutrients such as nitrogen and phosphorus [16,17]. Many potential pathogens of humans live in close association with plants and, therefore, have adapted to benefit plants in several ways in order to ensure their persistence. They can produce different vital phytohormones such as auxin, gibberellins and cytokinins. Some potentially pathogenic strains of Bacillus and Serratia are noted in the growth promotion of diverse crops such as potato, wheat and maize [18,19]. Therefore, the main objective of the present study was to evaluate the microbiological biosafety and agronomic significance of bacterial species associated with raw vegetables. For this purpose, enriched and selective culture media were used to isolate general and specific bacterial species associated with fresh produce. The final taxonomic status of the bacterial isolates was confirmed by 16S rRNA gene sequencing [20]. Fresh produce-associated microbial hazards have not been uncovered in detail in Pakistan. Therefore, in this study, bacterial diversity associated with carrot, cabbage and turnip was targeted. The main reason to work with these agronomic plants was their poor handling from field to local markets. This may have resulted in their cross contamination with potential human pathogens during transportation. It was also hypothesized that after interacting with host plants, bacterial species may have developed beneficial plant growth-promoting traits. With this in mind, the phylogenetic diversity and agricultural significance of bacterial communities were also inspected to identify the range of bacterial species showing positive interactions with colonized plants.

\section{Materials and Methods}

\subsection{Sample Collection and Culture Media}

Samples of carrot, cabbage and turnip (12 samples each) were collected from different sites in Lahore, Pakistan. Samples were collected from 12 randomly selected sites that were comprised of small vegetable shops, major vegetable markets and superstores. Samples were collected in sterile plastic bags and brought to the laboratory and processed within $24 \mathrm{~h}$. In order to assess the bacterial diversity, different types of culture media including Luria-Bertani Agar (L-agar), Baird-Parker Agar (BPA), Eosin Methylene Blue Agar (EMB), Mannitol Salt Agar (MSA), MacConkey Agar (MAC) and Xylose Lysine Deoxycholate Agar (XLD) were used. L-agar is extensively used as a general and non-selective growth medium [21]. BPA and MSA are selective for the growth of Gram-positive bacteria specifically for Staphylococcus aureus. EMB, MAC and XLD support the growth of Gram-negative bacteria. EMB agar, in particular, differentiates between lactose and non-lactose fermenter groups of Gram-negative bacteria and is widely used for the isolation of E. coli, Citrobacter freunddii and Enterobacter cloacae. 
XLD agar is particularly used for the isolation of Salmonella enterica and Shigella flexneri from food and clinical samples [22].

\subsection{Isolation of Bacterial Strains}

Samples were crushed in sterile mortars and pestles and diluted serially before spreading. For serial dilution, $10 \mathrm{~g}$ of the crushed sample was added into pre-autoclaved $100 \mathrm{~mL}$ normal saline solution $(0.85 \% \mathrm{NaCl})$ and mixed well. Approximately $50 \mu \mathrm{L}$ of this dilution was aseptically plated on the surface of L-agar and other selective media as mentioned above. After spreading, the plates were incubated between 30 to $37^{\circ} \mathrm{C}$ for $24 \mathrm{~h}$. Following incubation, colonies with different morphologies were selected and purified using several rounds of streaking on respective media (Figure S1). The purity of the colonies was also monitored by performing Gram staining.

\section{3. $16 \mathrm{~S}$ rRNA Gene Sequencing}

For $16 \mathrm{~S}$ rRNA gene sequencing, genomic DNA was extracted from bacterial cultures grown overnight using a Genomic DNA Purification Kit (Promega, USA). An approximately 1.5-kb DNA fragment containing $16 \mathrm{~S}$ rRNA gene was amplified using forward 27f and reverse primer 1522r [23]. PCR amplification was performed using $50 \mu \mathrm{L}$ of Dream Taq ${ }^{\mathrm{TM}}$ Green PCR Master Mix (ThermoFisher Scientific, Chelmsford, MA, USA) with $0.5 \mu \mathrm{g}$ of chromosomal DNA template and $0.5 \mu \mathrm{M}$ of each primer. The reaction mixtures were incubated in a thermocycler Primus 96 (PeQLab, Erlangen, Germany) at $94{ }^{\circ} \mathrm{C}$ for $5 \mathrm{~min}$ and passed through 30 cycles: denaturation for $20 \mathrm{~s}$ at $94{ }^{\circ} \mathrm{C}$, primer annealing for $20 \mathrm{~s}$ at $50{ }^{\circ} \mathrm{C}$ and extension at $72{ }^{\circ} \mathrm{C}$ for $2 \mathrm{~min}$. Final extension was carried out at $72{ }^{\circ} \mathrm{C}$ for $5 \mathrm{~min}$. The amplified products were purified using a QIAquick Gel Extraction Kit (QIAGEN, Frederick, MD, USA) and the samples were sent to First BASE laboratories (Singapore) for the sequencing of $16 \mathrm{~S}$ rRNA gene.

\subsection{Phylogenetic Analysis}

The sequences were analyzed using the bioinformatics tool, CHROMAS lite version 2.4.1.0, and homology was searched for in the NCBI Basic Local Alignment Search Tool (BLAST) for the identification of bacterial strains. After identification, all the sequences were aligned with a multiple sequence alignment program (ClustalW) using MEGA 6.05 software [24]. The phylogenetic relationships among different bacterial genera were studied after constructing phylogenetic trees using the neighbor-joining (NJ) method [25].

\subsection{Antibiotic Susceptibility Pattern of Bacterial Strains}

The antibiotic sensitivity pattern of purified bacterial strains was evaluated by following the method of Bauer et al. [26]. Plates of Mueller-Hinton agar were prepared and inoculated with bacterial strains using a sterile cotton swab to ensure confluent growth. The antibiotic susceptibility test discs (Bioanalyse ${ }^{\circledR}$, Ankara, Turkey) for gentamicin $(30 \mu \mathrm{g})$, tobramycin $(10 \mu \mathrm{g})$, amikacin $(30 \mu \mathrm{g})$, cephalexin $(30 \mu \mathrm{g})$, chloramphenicol $(30 \mu \mathrm{g})$, amoxicillin $(25 \mu \mathrm{g})$, nalidixic acid $(30 \mu \mathrm{g})$ and tetracycline $(30 \mu \mathrm{g})$ were aseptically placed to the surface of agar plates at well-spaced intervals. Three sets of plates for each strain or antibiotic were prepared for analysis. The plates were then incubated at $37^{\circ} \mathrm{C}$ for $24 \mathrm{~h}$. After incubation, the plates were observed for the presence of clear zones of inhibition around the antibiotic disc. Zones were measured in millimeters $(\mathrm{mm})$ using the Inhibition Zone Ruler provided by the manufacturer. The zones were then compared with the standardized chart for antibiotics (M100-S23) given by the clinical laboratory standard institute (CLSI, 2013).

\subsection{Functional Diversity of Plant-Associated Bacteria}

Production of auxin in $25 \mathrm{~mL}$ of Luria-Bertani broth (L-broth) medium was detected in the presence of 0 or $500 \mu \mathrm{g} \mathrm{mL} \mathrm{m}^{-1}$ of L-tryptophan. Flasks were inoculated with purified bacterial cultures 
in triplicate (adjusted to $10^{7} \mathrm{CFUs}$ per $\mathrm{mL}$ ) and incubated on a shaker in the dark at $37^{\circ} \mathrm{C}$ for $72 \mathrm{~h}$. After incubation, the cultures were centrifuged (Sigma 1-14, Osterode, Germany) at 18,900 relative centrifugal field (RCF) for $10 \mathrm{~min}$. One milliliter of supernatant was taken in a test tube and $2 \mathrm{~mL}$ of Salkowski's reagent was added [27]. The tubes were kept in the dark for $30 \mathrm{~min}$ for the development of pink to red coloration. Approximately $300 \mu \mathrm{L}$ of each sample was taken from the test tube and added into the wells of a microtiter plate. The intensity of the color was measured at $535 \mathrm{~nm}$ using a microplate spectrophotometer (Epoch, BioTek, Winooski, VT, USA). Finally, the concentrations of auxin produced by different isolates were determined by comparing the values with the standard curve. The standard curve was constructed using different concentrations of standard auxin ( 5 to $200 \mu \mathrm{g} \mathrm{mL} \mathrm{m}^{-1}$ ) in $1 \mathrm{~mL}$ of distilled water and processed for colorimetric analysis as mentioned above. Phosphate solubilization ability of the bacterial isolates was determined by streaking strains on Pikovskaya agar medium [28]. Hydrogen cyanide (HCN) activity of bacterial strains was determined as mentioned by Ahmad et al. [29].

\subsection{Biofilm Formation}

To determine the biofilm-forming ability of purified bacterial isolates the method by Christensen et al. [30] was followed with slight modifications. Bacterial strains were streaked on Tryptic Soy Agar (TSA) plates and incubated at $37^{\circ} \mathrm{C}$ for $24 \mathrm{~h}$. From the TSA plates, the cultures were picked and inoculated in Tryptic Soy Broth (TSB) medium and incubated as mentioned above. Following incubation, the broth cultures were standardized (optical density adjusted to 0.2 at $600 \mathrm{~nm}$ ) and $20 \mu \mathrm{L}$ of each culture was transferred to the wells of a 96-well flat bottom microtiter plate (Orange Scientific) that already contained $180 \mu \mathrm{L}$ of TSB medium. Negative controls with $200 \mu \mathrm{L}$ of TSB medium were also kept for comparison. The assay was performed in triplicate for all treatments. To promote biofilm formation, the plates were incubated aerobically on a shaker at $37^{\circ} \mathrm{C}$ for $72 \mathrm{~h}$. After incubation, the well contents were discarded and washed thrice with $250 \mu \mathrm{L}$ of sterile distilled water to remove any non-adherent and weakly adherent cells. Plates were air dried for $30 \mathrm{~min}$. The biofilm formed in the wells was fixed with $250 \mu \mathrm{L} /$ well of $98 \%$ methanol for $15 \mathrm{~min}$. After air drying, the fixed bacterial cells were stained with $200 \mu \mathrm{L}$ of $0.1 \% \mathrm{v} / \mathrm{v}$ crystal violet solution for $5 \mathrm{~min}$. The excessive stain was removed by placing the plate under slow running tap water and the plate was air dried. Re-solubilization of crystal violet with the adherent cells was done by adding $200 \mu \mathrm{L} /$ well of 33\% v/v glacial acetic acid. The optical density (OD) was measured at $570 \mathrm{~nm}$ using a microplate spectrophotometer (Epoch, BioTek, Winooski, VT, USA).

\subsection{Statistical Analysis}

Data for bacterial auxin production and biofilm formation was subjected to an analysis of variance (ANOVA) using SPSS 16 program. The means of different treatments were separated using Duncan's multiple range test $(p \leq 0.05)$.

\section{Results}

\section{1. $16 S$ rRNA Gene Sequencing}

The sequences obtained from First BASE laboratories were analyzed and refined by trimming the noise from both ends using the bioinformatics tool, CHROMAS lite version 2.4.1.0 (Sequences Supplementary file included). These sequences were used to search for homology with already identified sequences in NCBI using the default nucleotide Basic Local Alignment Search Tool (BLAST) settings. After comparison, the majority of the strains showed up to $99 \%$ similarity with their respective identified species. Analysis showed that the strains belong to Bacillus, Staphylococcus, Exiguobacterium, Lysinibacillus, Arthrobacter, Burkholderia, Acinetobacter, Pseudomonas, Stenotrophomonas, Serratia, Klebsiella, Citrobacter, Enterobacter, Pantoea and Kluyvera 
genera. After identification, the sequences were submitted in Genbank under accession numbers KJ865549 to KJ865603 (Tables 1-3).

Table 1. 16S rRNA gene sequencing of bacteria isolated from carrot at different temperatures and in different culture media.

\begin{tabular}{cccccc}
\hline $\begin{array}{c}\text { Serial } \\
\text { No. }\end{array}$ & Isolates & $\begin{array}{c}\text { Temperature } \\
\text { for Isolation }\end{array}$ & $\begin{array}{c}\text { Culture } \\
\text { Media }\end{array}$ & Identified as & Accessions \\
\hline 1 & BPc-4 & $30^{\circ} \mathrm{C}$ & L-agar & Bacillus cereus BPc-4 & KJ865556 \\
2 & EMc-3 & $37^{\circ} \mathrm{C}$ & L-agar & B. anthracis EMc-3 & KJ865553 \\
3 & LCw-22 & $30^{\circ} \mathrm{C}$ & L-agar & B. cereus LCw-22 & KJ865598 \\
4 & MSc-5 & $37^{\circ} \mathrm{C}$ & MSA & Staphylococcus warneri MSc-5 & KJ865590 \\
5 & Xc-7 & $30^{\circ} \mathrm{C}$ & L-agar & Lysinibacillus fusiformis Xc-7 & KJ865599 \\
6 & BPc-1 & $37^{\circ} \mathrm{C}$ & MAC & Serratia rubidaea BPc-1 & KJ865576 \\
7 & BPc-3 & $37^{\circ} \mathrm{C}$ & MAC & Pantoea dispersa BPc-3 & KJ865552 \\
8 & EMc-2 & $37^{\circ} \mathrm{C}$ & MAC & Se. rubidaea EMc-2 & KJ865581 \\
9 & EMc-4 & $37^{\circ} \mathrm{C}$ & L-agar & Acinetobacter calcoaceticus EMc-4 & KJ865567 \\
10 & Lc-52 & $37^{\circ} \mathrm{C}$ & L-agar & Ac. calcoaceticus Lc-52 & KJ865566 \\
11 & Lcr-22 & $37^{\circ} \mathrm{C}$ & MAC & Se. rubidaea Lcr-22 & KJ865575 \\
12 & Mc-2 & $37^{\circ} \mathrm{C}$ & MAC & Se. rubidaea Mc-2 & KJ865602 \\
13 & Mc-3 & $37^{\circ} \mathrm{C}$ & L-agar & Stenotrophomonas maltophilia Mc-3 & KJ865587 \\
14 & Mc-4 & $37^{\circ} \mathrm{C}$ & L-agar & Ac. calcoaceticus Mc-4 & KJ865588 \\
15 & MSc-1 & $37^{\circ} \mathrm{C}$ & MAC & Pantoea sp. MSc-1 & KJ865571 \\
16 & Xc-3 & $37^{\circ} \mathrm{C}$ & EMB & Enterobacter cloacae Xc-3 & KJ865572 \\
17 & Xc-5 & $30^{\circ} \mathrm{C}$ & L-agar & Pseudomonas putida Xc-5 & KJ865551 \\
18 & Xc-6 & $37^{\circ} \mathrm{C}$ & EMB & Citrobacter freundii Xc-6 & KJ865549 \\
\hline
\end{tabular}

Abbreviations: L-agar = Luria-Bertani Agar, MSA = Mannitol Salt Agar, MAC = MacConkey Agar, EMB = Eosine Methylene Blue agar.

Table 2. 16S rRNA gene sequencing of bacteria isolated from cabbage at different temperatures and in different culture media.

\begin{tabular}{|c|c|c|c|c|c|}
\hline $\begin{array}{l}\text { Serial } \\
\text { No. }\end{array}$ & Isolates & $\begin{array}{l}\text { Temperature } \\
\text { for Isolation }\end{array}$ & $\begin{array}{l}\text { Culture } \\
\text { Media }\end{array}$ & Identified as & Accessions \\
\hline 1 & $\mathrm{BPb}-5$ & $37^{\circ} \mathrm{C}$ & MSA & Staph. aureus $\mathrm{BPb}-5$ & KJ865591 \\
\hline 2 & $\mathrm{~Eb}-9$ & $30{ }^{\circ} \mathrm{C}$ & L-agar & B. cereus Eb-9 & KJ865594 \\
\hline 3 & $\mathrm{~Eb}-10$ & $30{ }^{\circ} \mathrm{C}$ & L-agar & B. thuringiensis Eb-10 & KJ865560 \\
\hline 4 & $\mathrm{Lb}-41$ & $30{ }^{\circ} \mathrm{C}$ & L-agar & Arthrobacter nicotianae Lb-41 & KJ865583 \\
\hline 5 & Lb-61 & $30{ }^{\circ} \mathrm{C}$ & L-agar & B. subtilis Lb-61 & KJ865595 \\
\hline 6 & $\mathrm{MCb}-3$ & $37^{\circ} \mathrm{C}$ & L-agar & Staph. arlettae $\mathrm{MCb}-3$ & KJ865592 \\
\hline 7 & $\mathrm{MCb}-4$ & $37^{\circ} \mathrm{C}$ & L-agar & Exiguobacterium mexicanum MCb-4 & KJ865577 \\
\hline 8 & MCb-6 & $30{ }^{\circ} \mathrm{C}$ & L-agar & B. cereus $\mathrm{MCb}-6$ & KJ865559 \\
\hline 9 & $\mathrm{MCb}-8$ & $30{ }^{\circ} \mathrm{C}$ & L-agar & B. subtilis $\mathrm{MCb}-8$ & KJ865584 \\
\hline 10 & MSb-3 & $30{ }^{\circ} \mathrm{C}$ & L-agar & B. cereus MSb-3 & KJ865596 \\
\hline 11 & MSb-4 & $37^{\circ} \mathrm{C}$ & L-agar & B. anthracis MSb-4 & KJ865558 \\
\hline 12 & $\mathrm{Xb}-6$ & $30{ }^{\circ} \mathrm{C}$ & L-agar & B. anthracis $\mathrm{Xb}-6$ & KJ865582 \\
\hline 13 & $\mathrm{BPb}-3$ & $37^{\circ} \mathrm{C}$ & L-agar & Ac. calcoaceticus $\mathrm{BPb}-3$ & KJ865562 \\
\hline 14 & $\mathrm{~Eb}-1$ & $37^{\circ} \mathrm{C}$ & MAC & Klebsiella pneumoniae Eb-1 & KJ865601 \\
\hline 15 & $\mathrm{~Eb}-2$ & $37^{\circ} \mathrm{C}$ & MAC & Pa. vagans $\mathrm{Eb}-2$ & KJ865561 \\
\hline 16 & $\mathrm{~Eb}-4$ & $30{ }^{\circ} \mathrm{C}$ & L-agar & Ac. calcoaceticus Eb-4 & KJ865586 \\
\hline 17 & $\mathrm{~Eb}-6$ & $30{ }^{\circ} \mathrm{C}$ & L-agar & Burkholderia cepacia Eb-6 & KJ865578 \\
\hline 18 & $\mathrm{~Eb}-8$ & $37^{\circ} \mathrm{C}$ & L-agar & Ac. calcoaceticus Eb-8 & KJ865568 \\
\hline 19 & $\mathrm{Xb}-3$ & $37^{\circ} \mathrm{C}$ & MAC & Se. rubidaea $\mathrm{Xb}-3$ & KJ865564 \\
\hline
\end{tabular}

Abbreviations: L-agar = Luria-Bertani Agar, MSA = Mannitol Salt Agar, MAC = MacConkey Agar, EMB = Eosine Methylene Blue agar. 
Table 3. 16S rRNA gene sequencing of bacteria isolated from turnip at different temperatures and in different culture media.

\begin{tabular}{|c|c|c|c|c|c|}
\hline $\begin{array}{l}\text { Serial } \\
\text { No. }\end{array}$ & Isolates & $\begin{array}{l}\text { Temperature } \\
\text { for Isolation }\end{array}$ & $\begin{array}{l}\text { Culture } \\
\text { Media }\end{array}$ & Identified as & Accessions \\
\hline 1 & BPt-5 & $37^{\circ} \mathrm{C}$ & MSA & Staph. equorum BPt-5 & KJ865579 \\
\hline 2 & Lt-41 & $37^{\circ} \mathrm{C}$ & MSA & Staph. xylosus Lt-41 & KJ865585 \\
\hline 3 & $\mathrm{Lt}-73$ & $37^{\circ} \mathrm{C}$ & MSA & Staph. warneri Lt-73 & KJ865565 \\
\hline 4 & MSt-1 & $37^{\circ} \mathrm{C}$ & MSA & Staph. xylosus MSt-1 & KJ865600 \\
\hline 5 & MSt-3 & $37^{\circ} \mathrm{C}$ & MSA & Staph. gallinarum MSt-3 & KJ865580 \\
\hline 6 & MSt-7 & $30^{\circ} \mathrm{C}$ & L-agar & B. cereus MSt-7 & KJ865557 \\
\hline 7 & MSt-8 & $30{ }^{\circ} \mathrm{C}$ & L-agar & B. cereus MSt- 8 & KJ865589 \\
\hline 8 & PCt-1 & $30{ }^{\circ} \mathrm{C}$ & L-agar & B. cereus PCt-1 & KJ865573 \\
\hline 9 & $X t-1$ & $37^{\circ} \mathrm{C}$ & MSA & Staph. xylosus Xt-1 & KJ865597 \\
\hline 10 & $X t-6$ & $30{ }^{\circ} \mathrm{C}$ & L-agar & L. fusiformis $\mathrm{Xt}-6$ & KJ865555 \\
\hline 11 & EMt-1 & $37^{\circ} \mathrm{C}$ & L-agar & Ac. bouvetii EMt-1 & KJ865593 \\
\hline 12 & EMt-5 & $37^{\circ} \mathrm{C}$ & EMB & E. amnigenus EMt-5 & KJ865563 \\
\hline 13 & MCt-1 & $37^{\circ} \mathrm{C}$ & L-agar & St. maltophilia MCt-1 & KJ865603 \\
\hline 14 & MCt-5 & $37^{\circ} \mathrm{C}$ & MAC & Kluyvera cryocrescens MCt-5 & KJ865554 \\
\hline 15 & MCt-6 & $37^{\circ} \mathrm{C}$ & MAC & Se. ureilytica MCt-6 & KJ865570 \\
\hline 16 & MSt-6 & $37^{\circ} \mathrm{C}$ & L-agar & Ac. calcoaceticus MSt- 6 & KJ865569 \\
\hline 17 & PCt-2 & $37^{\circ} \mathrm{C}$ & $\mathrm{EMB}$ & E. cloacae PCt-2 & KJ865574 \\
\hline 18 & $\mathrm{Xt}-3$ & $37^{\circ} \mathrm{C}$ & EMB & C. werkmannii $\mathrm{Xt}-3$ & KJ865550 \\
\hline
\end{tabular}

Abbreviations: L-agar = Luria-Bertani Agar, MSA = Mannitol Salt Agar, MAC = MacConkey Agar, EMB = Eosine Methylene Blue agar.

\subsection{Phylogenetic Analysis}

To study the phylogenetic relationships among bacterial strains, the sequences were first aligned with the multiple sequence alignment program (ClustalW) using MEGA 6 software. The phylogenetic tree was constructed using the neighbor-joining method to show evolutionary relationships among different isolates (Figure 1). In the phylogenetic tree, the first cluster was comprised of different strains of Bacillus. The second cluster includes Staphylococcus equorum BPt-5, Staph. gallinarum MSt-3, Staph. warneri MSc-5, Staph. arlettae MCb-3, Staph. xylosus Lt-41, Staph. aureus BPb-5, Staph. xylosus Xt-1, Staph. warneri Lt-73 and Staph. xylosus MSt-1. Similarly, different strains of Acinetobcter (Ac. bouvetii EMt-1, Ac. calcoaceticus MSt-6, Ac. calcoaceticus Mc-4, Ac. calcoaceticus Lc-52, Ac. colcoaceticus BPb-3, Ac. colcoaceticus Eb-4, Ac. colcoaceticus EMc-4, Ac. colcoaceticus Eb-8) covered another large cluster in the tree. Additionally, different strains of Gram-negative genera occupied the lower portion of the phylogenetic tree.

\subsection{Bacterial Diversity of Fresh Vegetables}

The microbiological analysis of carrot showed the association of 18 bacterial strains that belong to 11 bacterial genera (Table 1). The highest number for colonization was observed for the genera Serratia, Bacillus and Acinetobacter. For cabbage, 19 bacterial strains that represented nine bacterial genera were detected (Table 2). Maximum colonization was shown by the genus Bacillus followed by Acinetobacter and Staphylococcus. Additionally, some strains that specifically colonized cabbage include Ar. nicotianae Lb-41, Ex. mexicanum MCb-4, K. pneumoniae EB-1 and Bur. cepacia Eb-6. In the case of turnip, maximum colonization was shown by the genus Staphyloccous followed by Bacillus and Enterobacter (Table 3). Among the 18 strains that were detected from turnip, Kluyvera cryocrescens MCt-5 was specifically associated with this vegetable. Overall, the strains from the genus Bacillus, Staphylococcus, Serratia and Acinetobacter were frequently associated with the surfaces of carrot, turnip and cabbage, whereas, Lysinibacillus, Stenotrophomonas, Citrobacter and Enterobacter were commonly associated with carrot or turnip. Some bacterial genera were found to specifically colonize cabbage (Exiguobacterium, Arthrobacter, Burkholderia and Klebsiella), turnip (Kluyvera) or carrot (Pseudomonas and Pantoea). 


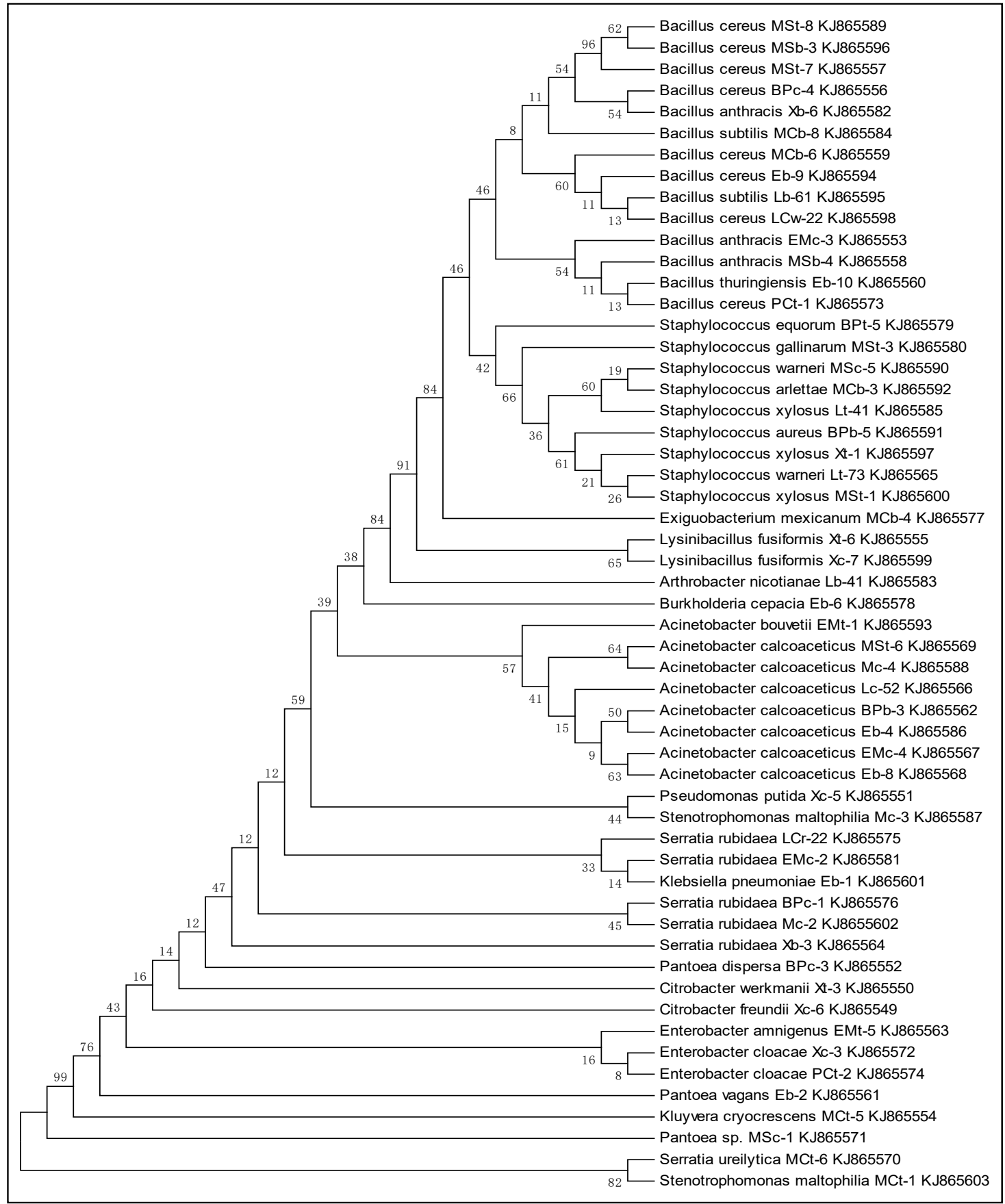

Figure 1. Phylogenetic analysis of 55 bacterial isolates associated with the surfaces of fresh vegetables (carrot, cabbage and turnip). Nucleotide sequences were trimmed after 16S rRNA gene sequencing. Phylogenies were inferred using the neighbor-joining method and the tree was constructed using MEGA 6 [24]. Numbers at the branch points indicate the percentage of 1000 bootstrap resampling.

\subsection{Antibiotic Susceptibility of Bacterial Strains}

The susceptibility of all the identified plant-associated bacterial isolates towards different antibiotics was determined. Figure 2 shows the sensitivity pattern of $B$. cereus LCw-22 and A. calcoaceticus MSt-6. Out of 55 strains, 30 and 27 strains were resistant against amoxicillin and nalidixic acid, respectively. Both of these drugs are broad-spectrum antibiotics which are equally effective for Gram-positive and Gram-negative groups of bacteria (Tables 4 and 5). Resistance against tobramycin, gentamicin and amikacin were shown by 18, eight and two bacterial strains, respectively. On the other 
hand, 24 bacterial strains were resistant to cephalexin. Chloramphenicol and tetracycline are also broad-spectrum antibiotics and resistance against them was depicted, respectively, by seven and five strains while others recorded sensitive or intermediate results. For Gram-positive bacteria, the majority of the strains recorded sensitivity against amikacin, gentamicin, tetracycline and chloramphenicol (Table 4). St. maltophilia Mc-3 showed the highest resistance against the applied antibiotics as out of eight, it recorded resistant against seven antibiotics (Table 5). Similarly, B. subtilis MCb-8, Ent. cloacae PCt-2, Ent. amnigenus EMt-5 and Staph. warneri Lt-73 showed resistance for six, five, five and five antibiotics, respectively.

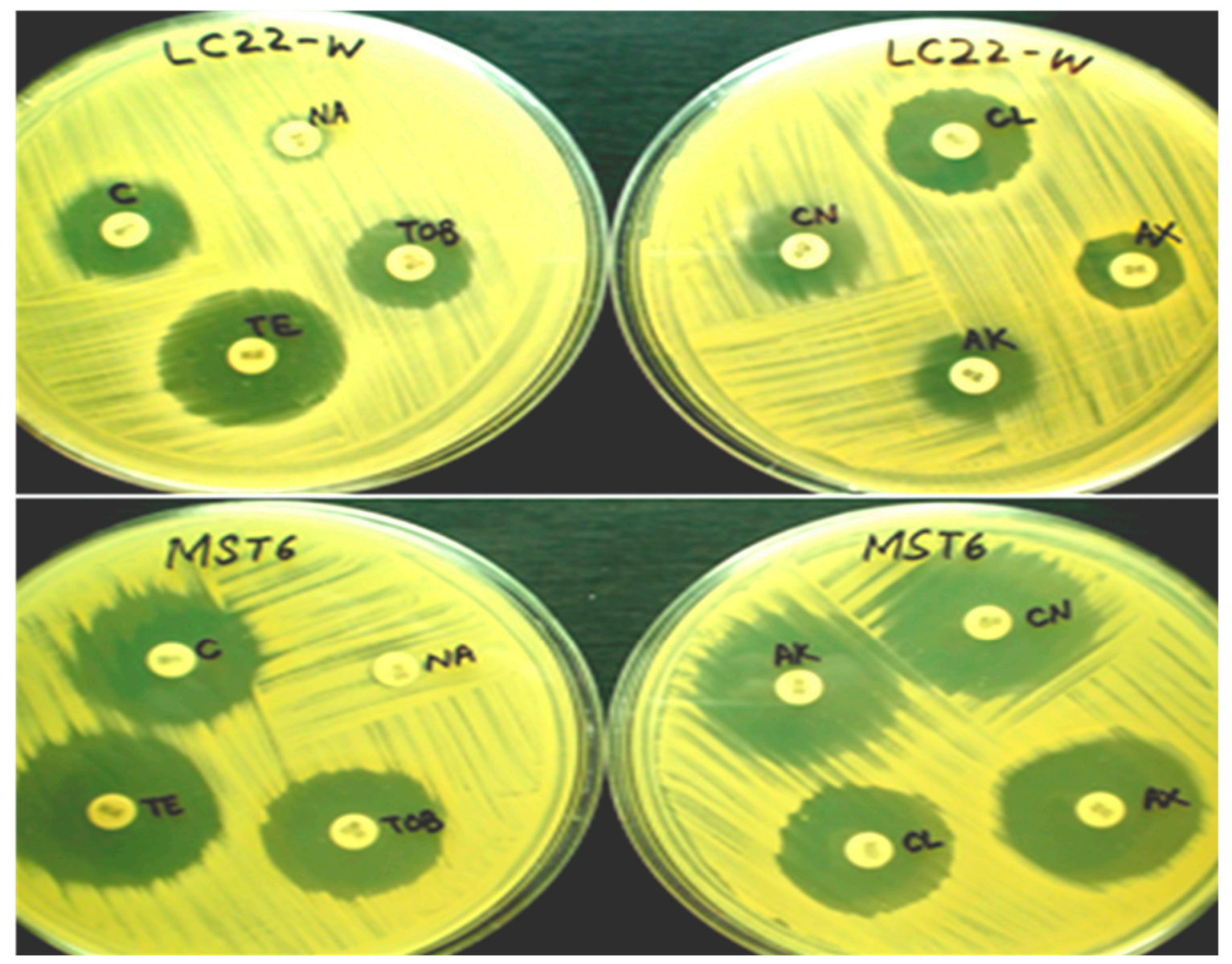

Figure 2. Antibiotic susceptibility pattern of purified strains of B. cereus LCw-22 and A. calcoaceticus MSt-6. Plates were incubated at $37^{\circ} \mathrm{C}$ for $24 \mathrm{~h}$. Abbreviations: $\mathrm{C}=$ chloramphenicol, $\mathrm{NA}=$ nalidixic acid, $\mathrm{TE}=$ tetracycline, $\mathrm{TOB}=$ tobramycin, $\mathrm{AK}=$ amikacin, $\mathrm{CN}=$ gentamicin, $\mathrm{CL}=$ cephalexin, $\mathrm{AX}=$ amoxicillin.

\subsection{Functional Diversity of Bacteria}

Bacterial isolates were evaluated for their auxin production ability in the presence of $500 \mu \mathrm{g} \mathrm{mL}-1$ L-tryptophan. The highest levels of auxin were detected for B. cereus PCt-1, B. cereus MSb-3 and Se. ureilytica MCt-6, which recorded 5-, 4- and 4-fold increases, respectively, compared to the un-amended control. A significant 3-fold increase was observed with each of the strains: Pan. vagans Eb-2, Se. rubidaea EMc-2, Ac. calcoaceticus Eb-4, Se. rubidaea Mc-2 and Cit. freundii Xc-6. The maximum auxin concentrations of 36,34 and $33 \mu \mathrm{g} \mathrm{mL} \mathrm{mL}^{-1}$ were obtained by L. fusiformis Xt-6, Ent. cloacae PCt-2 and K. pneumoniae Eb-1, respectively, in the absence of L-tryptophan. However, in the L-tryptophan-amended medium, St. maltophilia MCt-1 $\left(101 \mu \mathrm{g} \mathrm{mL}{ }^{-1}\right)$, B. cereus PCt-1 (97 $\left.\mu \mathrm{g} \mathrm{mL}{ }^{-1}\right)$, Se. rubidaea Mc-2 $\left(77 \mu \mathrm{g} \mathrm{mL}{ }^{-1}\right)$, K. pneumoniae Eb-1 $\left(75 \mu \mathrm{g} \mathrm{mL}^{-1}\right)$ and Ent. cloacae PCt-2 $\left(71 \mu \mathrm{g} \mathrm{mL} \mathrm{m}^{-1}\right)$ were the most promising for in vitro auxin production (Figure 3). 


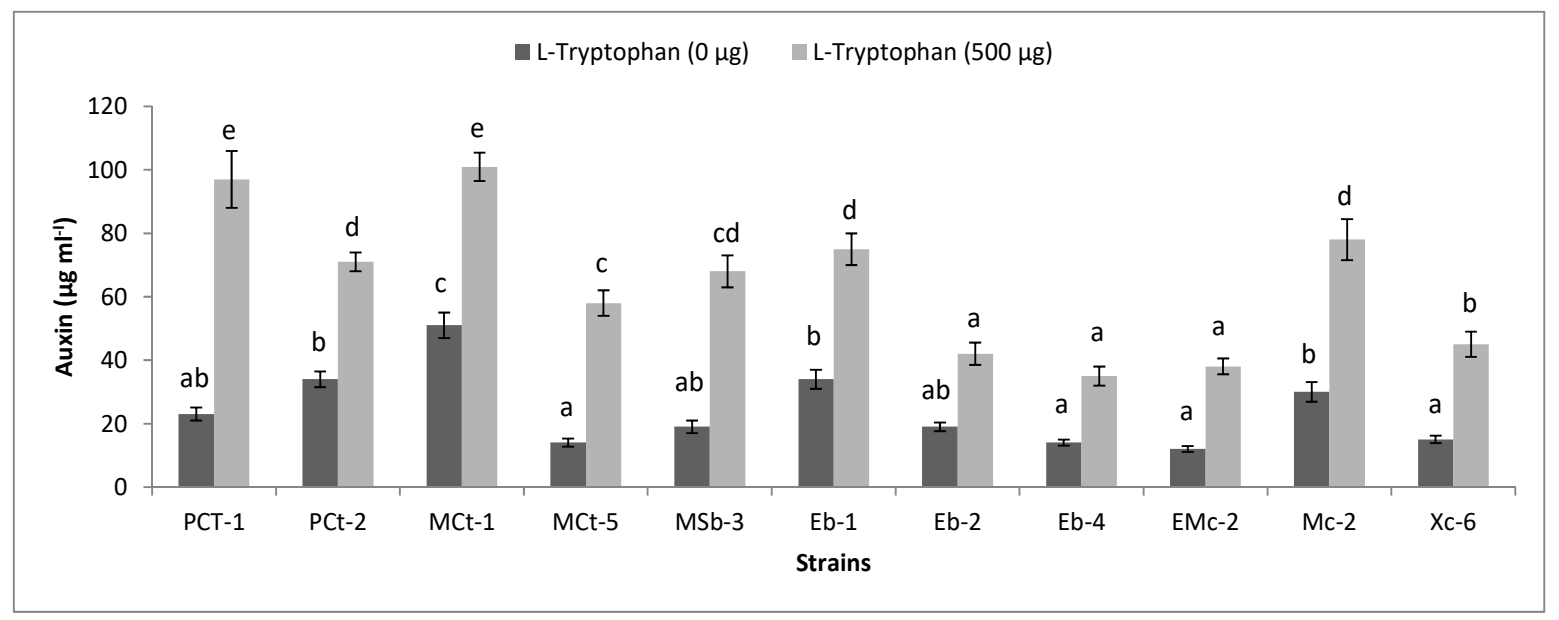

Figure 3. Auxin production by some representative purified bacterial strains in the presence and absence of L-tryptophan. Strains were grown in Luria-Bertani broth (L-broth) at $37^{\circ} \mathrm{C}$ for $72 \mathrm{~h}$. Bar represents the mean \pm S.E. of three replicates. Different letters on bars indicate significant differences between respective treatments using Duncan's multiple range test $(p \leq 0.05)$.

Table 4. Antibiotic susceptibility pattern of different Gram-positive bacterial isolates. For analysis, three set of plates for each strain or antibiotic were incubated at $37^{\circ} \mathrm{C}$ for $24 \mathrm{~h}$.

\begin{tabular}{|c|c|c|c|c|c|c|c|c|}
\hline \multirow{3}{*}{ Strains } & \multicolumn{8}{|c|}{ Antibiotics } \\
\hline & AK & $\mathrm{AX}$ & CL & $\mathrm{CN}$ & NA & ТОВ & TE & $\mathrm{C}$ \\
\hline & \multicolumn{8}{|c|}{ Zones of Inhibition $(\mathrm{mm}) *$} \\
\hline Bacillus cereus BPc- 4 & $18(S)$ & $10(\mathrm{R})$ & $8(\mathrm{R})$ & $14(\mathrm{I})$ & $14(\mathrm{I})$ & $15(S)$ & $22(S)$ & $10(\mathrm{R})$ \\
\hline Staphylococcus equorum BPt-5 & $26(S)$ & $24(\mathrm{R})$ & $11(\mathrm{R})$ & $20(S)$ & $0(\mathrm{R})$ & $16(S)$ & $16(\mathrm{I})$ & $26(S)$ \\
\hline S. aureus $\mathrm{BPb}-5$ & $18(S)$ & $8(\mathrm{R})$ & $18(S)$ & $18(S)$ & $14(\mathrm{I})$ & $16(S)$ & $26(S)$ & $24(S)$ \\
\hline B. anthracis EMc-3 & $20(\mathrm{~S})$ & $16(\mathrm{I})$ & $14(\mathrm{I})$ & $24(S)$ & $16(\mathrm{I})$ & $24(\mathrm{~S})$ & $24(S)$ & $24(S)$ \\
\hline B. cereus Eb-9 & $16(S)$ & $20(S)$ & $0(\mathrm{R})$ & $22(S)$ & $20(S)$ & $12(\mathrm{R})$ & $18(\mathrm{I})$ & $24(S)$ \\
\hline B. thuringiensis Eb-10 & $18(\mathrm{~S})$ & $20(S)$ & $0(\mathrm{R})$ & $14(\mathrm{I})$ & $0(\mathrm{R})$ & $12(\mathrm{R})$ & $20(S)$ & $12(\mathrm{R})$ \\
\hline S. $x y$ losus Lt-41 & $20(\mathrm{~S})$ & $24(\mathrm{R})$ & $22(\mathrm{~S})$ & $20(S)$ & $0(\mathrm{R})$ & $18(S)$ & $22(S)$ & $20(S)$ \\
\hline S. warneri Lt-73 & $20(S)$ & $0(\mathrm{R})$ & $0(\mathrm{R})$ & $12(\mathrm{R})$ & $0(\mathrm{R})$ & $10(\mathrm{R})$ & $16(\mathrm{I})$ & $24(S)$ \\
\hline Arthrobacter nicotianae Lb-41 & $22(S)$ & $26(S)$ & $16(S)$ & $16(S)$ & $0(\mathrm{R})$ & $14(\mathrm{I})$ & $20(S)$ & $30(S)$ \\
\hline B. subtilis Lb-61 & $28(S)$ & $20(S)$ & $14(\mathrm{I})$ & $30(S)$ & $12(\mathrm{R})$ & $22(S)$ & $26(S)$ & $14(\mathrm{I})$ \\
\hline B. cereus LCw-22 & $16(\mathrm{I})$ & $14(\mathrm{I})$ & $20(\mathrm{~S})$ & $16(S)$ & $10(\mathrm{R})$ & $16(\mathrm{~S})$ & $24(S)$ & $18(\mathrm{~S})$ \\
\hline S. arlettae $\mathrm{MCb}-3$ & $32(\mathrm{~S})$ & $14(\mathrm{I})$ & $22(\mathrm{~S})$ & $28(S)$ & $0(\mathrm{R})$ & $22(\mathrm{~S})$ & $8(\mathrm{R})$ & $24(S)$ \\
\hline Exiguobacterium mexicanum MCb-4 & $22(\mathrm{~S})$ & $38(S)$ & $28(S)$ & $20(S)$ & $18(\mathrm{I})$ & $16(S)$ & $26(S)$ & $24(S)$ \\
\hline B. cereus $\mathrm{MCb}-6$ & $20(\mathrm{~S})$ & $14(\mathrm{I})$ & $18(\mathrm{~S})$ & $16(S)$ & $0(\mathrm{R})$ & $14(\mathrm{I})$ & $24(S)$ & $16(\mathrm{I})$ \\
\hline B. subtilis $\mathrm{MCb}-8$ & $16(\mathrm{I})$ & $0(\mathrm{R})$ & $0(\mathrm{R})$ & $10(\mathrm{R})$ & $12(\mathrm{R})$ & $10(\mathrm{R})$ & $16(\mathrm{I})$ & $10(\mathrm{R})$ \\
\hline S. xylosus MSt-1 & $24(\mathrm{~S})$ & $22(\mathrm{R})$ & $10(\mathrm{R})$ & $20(S)$ & $0(\mathrm{R})$ & $20(S)$ & $20(S)$ & $24(S)$ \\
\hline S. gallinarum MSt-3 & $14(\mathrm{I})$ & $12(\mathrm{R})$ & $16(S)$ & $14(\mathrm{I})$ & $0(\mathrm{R})$ & $10(\mathrm{R})$ & $20(S)$ & $18(S)$ \\
\hline B. cereus MSt-7 & $26(S)$ & $14(\mathrm{I})$ & $32(S)$ & $24(S)$ & $18(\mathrm{I})$ & $18(S)$ & $14(\mathrm{R})$ & $20(S)$ \\
\hline B. cereus MSt- 8 & $18(\mathrm{~S})$ & $8(\mathrm{R})$ & $14(\mathrm{I})$ & $12(\mathrm{R})$ & $16(\mathrm{I})$ & $18(\mathrm{~S})$ & $24(S)$ & $28(S)$ \\
\hline B. cereus MSb-3 & $34(S)$ & $42(S)$ & $24(S)$ & $30(S)$ & $12(\mathrm{R})$ & $18(S)$ & $24(S)$ & $30(S)$ \\
\hline B. anthracis MSb-4 & $14(\mathrm{I})$ & $16(\mathrm{I})$ & $12(\mathrm{I})$ & $14(\mathrm{I})$ & $14(\mathrm{I})$ & $20(S)$ & $18(\mathrm{I})$ & $20(S)$ \\
\hline S. warneri MSc-5 & $24(S)$ & $40(S)$ & $38(S)$ & $30(S)$ & $14(\mathrm{I})$ & $24(\mathrm{~S})$ & $28(S)$ & $24(S)$ \\
\hline B. cereus PCt-1 & $18(\mathrm{~S})$ & $0(\mathrm{R})$ & $0(\mathrm{R})$ & $14(\mathrm{I})$ & $16(\mathrm{I})$ & $12(\mathrm{R})$ & $16(\mathrm{I})$ & $16(\mathrm{I})$ \\
\hline S. $x y$ losus $\mathrm{Xt}-1$ & $20(\mathrm{~S})$ & $20(\mathrm{R})$ & $20(S)$ & $18(S)$ & $0(\mathrm{R})$ & $18(\mathrm{~S})$ & $22(S)$ & $20(S)$ \\
\hline Lysinibacillus fusiformis $\mathrm{Xt}-6$ & $16(\mathrm{I})$ & $0(\mathrm{R})$ & $0(\mathrm{R})$ & $14(\mathrm{I})$ & $14(\mathrm{I})$ & $12(\mathrm{R})$ & $18(\mathrm{I})$ & $20(S)$ \\
\hline B. anthracis $\mathrm{Xb}-6$ & $22(S)$ & $12(\mathrm{R})$ & $14(\mathrm{I})$ & $16(S)$ & $12(\mathrm{R})$ & $16(S)$ & $20(S)$ & $20(S)$ \\
\hline L. fusiformis $\mathrm{Xc}_{\mathrm{c}-7}$ & $34(\mathrm{~S})$ & $0(\mathrm{R})$ & $0(\mathrm{R})$ & $25(S)$ & $0(\mathrm{R})$ & $8(\mathrm{R})$ & $32(S)$ & $40(S)$ \\
\hline
\end{tabular}

${ }^{*}$ Letters in parenthesis indicate the level of sensitivity of the respective antibiotics. Abbreviations: $\mathrm{R}=$ resistant, $\mathrm{I}=$ intermediate, $\mathrm{S}=$ sensitive. Antibiotics: $\mathrm{AK}=$ Amikacin; $\mathrm{AX}=$ Amoxicillin; $\mathrm{CL}=$ Cephalexin; $\mathrm{CN}=$ Gentamicin; $\mathrm{NA}$ $=$ Nalidixic acid $; \mathrm{TOB}=$ Tobramycin; $\mathrm{TE}=$ Tetracycline $; \mathrm{C}=$ Chloramphenicol . 
Table 5. Antibiotic susceptibility pattern of different Gram-negative bacterial isolates. For analysis, three set of plates for each strain or antibiotic were incubated at $37^{\circ} \mathrm{C}$ for $24 \mathrm{~h}$.

\begin{tabular}{|c|c|c|c|c|c|c|c|c|}
\hline \multirow{3}{*}{ Strains } & \multicolumn{8}{|c|}{ Antibiotics } \\
\hline & AK & $A X$ & CL & $\mathrm{CN}$ & NA & TOB & TE & $\mathrm{C}$ \\
\hline & \multicolumn{8}{|c|}{ Zone of Inhibition (mm)* } \\
\hline Serratia rubidaea BPc-1 & $18(S)$ & $12(\mathrm{R})$ & $0(\mathrm{R})$ & $14(\mathrm{I})$ & $14(\mathrm{I})$ & $12(\mathrm{R})$ & 14 (R) & $18(S)$ \\
\hline Pantoea dispersa BPc-3 & $22(S)$ & $14(\mathrm{I})$ & $14(\mathrm{I})$ & $18(\mathrm{~S})$ & $12(\mathrm{R})$ & $14(\mathrm{I})$ & $20(S)$ & $12(\mathrm{R})$ \\
\hline Acinetobacter calcoaceticus $\mathrm{BPb}-3$ & $16(\mathrm{I})$ & $14(\mathrm{I})$ & $12(\mathrm{I})$ & $18(\mathrm{~S})$ & $14(\mathrm{I})$ & $20(S)$ & $26(S)$ & $22(S)$ \\
\hline Klebsiella penumoniae Eb-1 & $18(S)$ & $0(\mathrm{R})$ & $14(\mathrm{I})$ & $18(\mathrm{~S})$ & $18(\mathrm{I})$ & $12(\mathrm{R})$ & $20(S)$ & $22(S)$ \\
\hline P. vagans Eb-2 & $20(S)$ & $22(S)$ & $20(S)$ & $20(S)$ & $18(\mathrm{I})$ & $22(S)$ & $22(S)$ & $10(\mathrm{R})$ \\
\hline A. calcoaceticus Eb-4 & $20(S)$ & $0(\mathrm{R})$ & $10(\mathrm{R})$ & $12(\mathrm{R})$ & $14(\mathrm{I})$ & $16(\mathrm{~S})$ & $18(\mathrm{I})$ & $20(S)$ \\
\hline Burkholderia cepacia Eb-6 & $24(S)$ & $16(\mathrm{I})$ & $20(S)$ & $18(S)$ & $0(\mathrm{R})$ & $20(S)$ & $20(S)$ & $14(\mathrm{I})$ \\
\hline A. calcoaceticus $\mathrm{Eb}-8$ & $18(\mathrm{~S})$ & $8(\mathrm{R})$ & $0(\mathrm{R})$ & $16(S)$ & $16(\mathrm{I})$ & $14(\mathrm{I})$ & $20(S)$ & $12(\mathrm{R})$ \\
\hline A. bouvetii EMt-1 & $18(\mathrm{~S})$ & $22(S)$ & $26(S)$ & $14(\mathrm{I})$ & $18(\mathrm{I})$ & $24(\mathrm{~S})$ & $26(S)$ & $22(S)$ \\
\hline Enterobacter amnigenus EMt-5 & $18(\mathrm{~S})$ & $0(\mathrm{R})$ & $0(\mathrm{R})$ & $12(\mathrm{R})$ & $12(\mathrm{R})$ & $10(\mathrm{R})$ & $16(\mathrm{I})$ & $22(S)$ \\
\hline S. rubidaea EMc-2 & $26(S)$ & $12(\mathrm{R})$ & $16(S)$ & $16(S)$ & $0(\mathrm{R})$ & $14(\mathrm{I})$ & $22(\mathrm{~S})$ & $16(\mathrm{I})$ \\
\hline A. calcoaceticus EMc-4 & $28(S)$ & $14(\mathrm{I})$ & $22(\mathrm{~S})$ & $16(S)$ & $16(\mathrm{I})$ & $18(S)$ & $18(\mathrm{I})$ & $20(S)$ \\
\hline A. calcoaceticus Lc-52 & $16(\mathrm{I})$ & $12(\mathrm{R})$ & $12(\mathrm{I})$ & $14(\mathrm{I})$ & $12(\mathrm{R})$ & $14(\mathrm{I})$ & $18(\mathrm{I})$ & $18(S)$ \\
\hline S. rubidaea Lcr- 22 & $22(S)$ & $14(\mathrm{I})$ & $0(\mathrm{R})$ & $16(\mathrm{~S})$ & $18(\mathrm{I})$ & $12(\mathrm{R})$ & $18(\mathrm{I})$ & $20(S)$ \\
\hline Stenotrophomonas maltophilia MCt-1 & $16(\mathrm{I})$ & $0(\mathrm{R})$ & $0(\mathrm{R})$ & $14(\mathrm{I})$ & $22(S)$ & $10(\mathrm{R})$ & $20(S)$ & $22(S)$ \\
\hline Kluyvera cryocrescens MCt-5 & $12(\mathrm{R})$ & $10(\mathrm{R})$ & $10(\mathrm{R})$ & $14(\mathrm{I})$ & $12(\mathrm{R})$ & $16(S)$ & $18(\mathrm{I})$ & $18(\mathrm{~S})$ \\
\hline S. ureilytica MCt-6 & $14(\mathrm{I})$ & $10(\mathrm{R})$ & $14(\mathrm{I})$ & $12(\mathrm{R})$ & $14(\mathrm{I})$ & $16(S)$ & $22(S)$ & $20(S)$ \\
\hline S. rubidaea Mc-2 & $24(\mathrm{~S})$ & $18(S)$ & $14(\mathrm{I})$ & $16(S)$ & $12(\mathrm{R})$ & $18(S)$ & $16(\mathrm{I})$ & $22(S)$ \\
\hline S. maltophilia Mc-3 & $16(\mathrm{I})$ & $0(\mathrm{R})$ & $0(\mathrm{R})$ & $10(\mathrm{R})$ & $0(\mathrm{R})$ & $0(\mathrm{R})$ & $10(\mathrm{R})$ & $12(\mathrm{R})$ \\
\hline A. calcoaceticus Mc-4 & $26(S)$ & $20(S)$ & $28(S)$ & $28(S)$ & $0(\mathrm{R})$ & $26(S)$ & $20(S)$ & $28(S)$ \\
\hline A. calcoaceticus MSt-6 & $28(S)$ & $26(S)$ & $24(S)$ & $28(S)$ & $0(\mathrm{R})$ & $24(S)$ & $28(\mathrm{~S})$ & $24(S)$ \\
\hline Pantoea sp. MSc-1 & $28(S)$ & $20(S)$ & $18(S)$ & $22(S)$ & $20(S)$ & $25(S)$ & $26(S)$ & $18(S)$ \\
\hline E. cloacae PCt-2 & $12(\mathrm{R})$ & $14(\mathrm{I})$ & $10(\mathrm{R})$ & $10(\mathrm{R})$ & $0(\mathrm{R})$ & $10(\mathrm{R})$ & $18(\mathrm{I})$ & $18(S)$ \\
\hline E. cloacae $\mathrm{Xc}_{\mathrm{c}-3}$ & $18(\mathrm{~S})$ & $0(\mathrm{R})$ & $0(\mathrm{R})$ & $16(S)$ & $20(S)$ & $10(\mathrm{R})$ & $20(\mathrm{~S})$ & $22(S)$ \\
\hline Pseudomonas putida $\mathrm{Xc}_{\mathrm{c}-5}$ & $34(\mathrm{~S})$ & $12(\mathrm{R})$ & $10(\mathrm{R})$ & $28(S)$ & $16(\mathrm{I})$ & $30(S)$ & $36(S)$ & $16(\mathrm{I})$ \\
\hline Citrobacter freundii $\mathrm{X}_{\mathrm{c}-} 6$ & $18(\mathrm{~S})$ & $10(\mathrm{R})$ & $0(\mathrm{R})$ & $16(S)$ & $14(\mathrm{I})$ & $10(\mathrm{R})$ & $14(\mathrm{R})$ & $20(S)$ \\
\hline C. werkmannii Xt-3 & $16(\mathrm{I})$ & $0(\mathrm{R})$ & $0(\mathrm{R})$ & $18(\mathrm{~S})$ & $16(\mathrm{I})$ & $12(\mathrm{R})$ & $18(\mathrm{I})$ & $20(S)$ \\
\hline S. rubidaea $\mathrm{Xb}-3$ & $22(\mathrm{~S})$ & $12(\mathrm{R})$ & $0(\mathrm{R})$ & $18(\mathrm{~S})$ & $22(\mathrm{~S})$ & $14(\mathrm{I})$ & $16(\mathrm{I})$ & $22(\mathrm{~S})$ \\
\hline
\end{tabular}

${ }^{*}$ Letters in parenthesis indicate level of sensitivity of respective antibiotics. Affiliations: $\mathrm{R}=$ resistant, $\mathrm{I}=$ intermediate, $\mathrm{S}=$ sensitive. Antibiotics: $\mathrm{AK}=$ Amikacin; $\mathrm{AX}=$ Amoxicillin; $\mathrm{CL}=$ Cephalexin; $\mathrm{CN}=$ Gentamicin; NA

$=$ Nalidixic acid $; \mathrm{TOB}=$ Tobramycin; $\mathrm{TE}=$ Tetracycline $; \mathrm{C}=$ Chloramphenicol.

For HCN production, Pan. dispersa BPc-3 and B. subtilis Lb-61 were strongly positive and turned the color of the filter paper to a dark orange (Figure S2). Similarly, Ac. calcoaceticus Eb-4, Ac. calcoaceticus Eb-8, Ac. calcoaceticus BPb-3, K. pneumoniae Eb-1, B. subtilis MCb-8 and B. cereus MSb-3 produced clear zones after inoculation on Pikovskaya media, which indicated a positive test for phosphate solubilization (Figure S3).

\subsection{Biofilm Formation}

The biofilm-forming potential of purified bacterial isolates was examined by a microtiter plate assay. After $72 \mathrm{~h}$ of incubation at $37^{\circ} \mathrm{C}$ in a 96-well microtiter plate, the cells were stained with crystal violet and the cell biomass was recorded by a spectrophotometer. The strains giving a cell mass OD equal to or above 1 were considered good biofilm producers (Figure 4). The bacterial strains Ar. nicotianae Lb-41, Staph. arlettae MCb-3, Ex. mexicanum MCb-4 and Staph. xylosus Xt-1 were observed as strong biofilm producers. Similarly, good biofilm production was also noted with K. pneumoniae Eb-1, Ac. calcoaceticus Eb-8, Se. rubidaea LCr-22, Staph. xylosus Lt-41, B. cereus LCw-22, Kl. ryocrescens MCt-5, Se. rubidaea Mc-2, B. anthracis MSb-4 and Cit. werkmanii Xt-3. The bacterial strains also showed variations in their ability to form an in vitro biofilm. For instance, K. pneumoniae Eb-1, Ac. calcoaceticus Eb-8, B. anthracis MSb-4 and A. nicotianae Lb-41 that were associated with cabbage recorded good potential for biofilm formation as compared to other crop isolates. For carrot, three bacterial strains 
that include B. cereus LCw-22 and Se. rubidaea (Mc-2, Lcr-22) showed significant biofilm formation. In the case of turnip, Staph. xylosus (Lt-41, Xt-1) was found to be very effective as a biofilm producer.

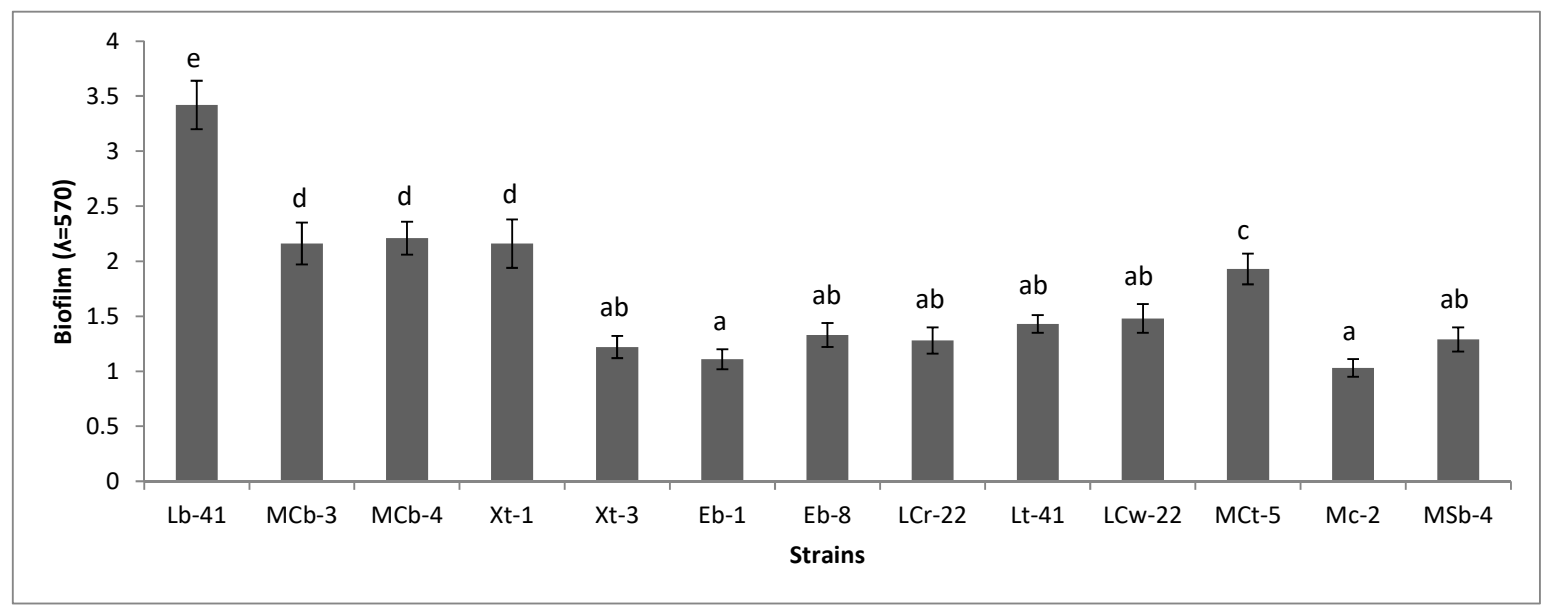

Figure 4. Biofilm formation by some selected purified bacterial strains. Biofilm assay was performed in Tryptic Soy Broth (TSB) medium at $37^{\circ} \mathrm{C}$. Bar represents the mean \pm S.E. of three replicates. Different letters on bars indicates significant differences between treatments using Duncan's multiple range test $(p \leq 0.05)$.

\section{Discussion}

Fresh vegetables are colonized by thousands of microbial species which may or may not be a part of their natural microbiome. Extraneous microorganisms can be found associated with plants due to contamination from different sources such as unclean irrigation water, organic fertilizers, animal and human wastes [10]. If pathogenic, these microorganisms can influence human health in several ways and thus present serious food safety challenges. A number of studies have already explored fresh vegetables for specific plant-associated human pathogens [31-33]. The present study demonstrated the biosafety concerns associated with raw-eaten fresh vegetables from some areas of Lahore, Pakistan. Moreover, little is known about the microbiological hazards associated with fresh agricultural produce in Pakistan. Therefore, in the present work, we focused on the sanitation of local vegetable markets with concurrent screening for beneficial bacteria-plant interactions.

Although no obligate human pathogens were detected in our vegetable samples, some potentially pathogenic bacteria that were isolated include B. cereus, B. anthracis, Staph. aurues, Ent. cloacae, Ent. amnigenus and K. Pneumoniae. The presence of such organisms in fresh raw-eaten vegetables is a serious concern for the consumer's health. B. cereus is a common foodborne human pathogen that causes food poisoning by producing toxins in food. It has been reported to be present in the intestinal tract of mammals and their waste material [34]. B. cereus can cause two distinct types of food poisoning that includes diarrheal or emetic syndrome. Nonhemolytic enterotoxin has been shown to be associated with diarrheal syndrome. It is also responsible for a variety of local and systemic infections [35]. Ent. cloacae, Ent. amnigenus and K. Pneumoniae are members of the Enterobacteriaceae family and their incidence in vegetables is also linked to the fecal material of warm-blooded animals. Enterobacter is also a member of the coliform group of bacteria which share their growth properties with human pathogenic bacteria. Their presence clearly indicates the possible presence of pathogens in these vegetables and their consumption without proper precautions can impact human health badly. A study by Falomir et al. [36] also reported the isolation of Ent. cloacae and K. pneumoniae from fresh vegetables. Staphylococcal enterotoxins (SEs) are a major cause of food poisoning, which typically occurs after ingestion of different contaminated food products. It has been shown that SEA and SEH enterotoxins are the most common cause of staphylococcal food poisoning around the world [37]. B. cereus, E. coli, Listeria monocytogenes and Salmonella are considered to be the most frequent bacterial 
pathogens associated with fresh produce-related outbreaks [38]. In most of the cases, contaminated vegetables with the members of Enterobacteriaceae were associated with gastrointestinal diseases such as diarrhea [39]. Similarly, produce-related pathogenic organisms such as Clostridium botulinum, B. cereus and S. aureus produce heavy amounts of toxins during their colonization and are the major causes of food poisoning [40].

As the isolation of some potential pathogens from fresh vegetables made the safety of these products questionable, it was necessary to screen all the identified microorganisms for multidrug resistance. High resistances of $52 \%$ (amoxicillin) and $59 \%$ (nalidixic acid) were observed with two broad-spectrum antibiotics used for Gram-positive bacteria (Table 4). Antibiotics used against Gram-negative bacteria were effective for the majority of the strains (Table 5). However, $57 \%$ and $50 \%$ resistance was recorded for amoxicillin and cephalexin, respectively. In the present study, a few strains of B. cereus (LCw-22, MSt-7, MSb-3) and Pantoea sp. MSc-1 were sensitive to the tested drugs. This may show the potential of these drugs in bacterial disease prevention. Nevertheless, multiple resistance against amoxicillin, tetracycline, gentamicin, chloamphenicol and other chemotherapeutic agents have been reported in Enterobacter and Klebsiella species isolated from fresh vegetables [36].

In this study, bacterial strains associated with fresh agricultural produce also exhibited beneficial plant growth-promoting attributes; especially, auxin production and biofilm formation. A variety of IAA-producing bacterial strains has been shown to harbor by plants that positively influence plant growth and productivity [41-44]. In the present study, supplementation of L-broth with L-tryptophan enhanced auxin production several folds compared to the control. For instance, Se. rubidaea EMc-2 recorded the lowest production of auxin $\left(12.24 \mu \mathrm{g} \mathrm{mL}^{-1}\right)$ in the absence of L-tryptophan. However, supplementation of the medium with L-tryptophan resulted in a 2-fold increase in auxin content in liquid culture supernatants (Table S1). L-tryptophan has been considered as precursor for auxin biosynthesis in plants as well as for microbes [20]. The results of present study may indicate the ability of microbes to use the natural source of L-tryptophan from root exudates or soil to produce phytohormones within the plant's rhizosphere. In this way, microbes may provide an exogenous source of phytohormones to plants, especially during the early stages of development [45]. A variety of bacterial genera can interact and colonize plant surfaces by the formation of biofilms. Biofilm formation on plant surfaces may be associated with symbiotic or pathogenic response depending on the microbial species [46]. Our results showed the colonization of fresh agricultural produce by a few potential human pathogens that belong to the genera of Bacillus, Enterobacter, Staphylococcus and Klebsiella. In present study, K. pneumonia Eb-1 and B. anthracis MSb-1 that were associated with cabbage showed significant biofilm formation (Figure 4). Thus, these opportunistic human pathogens can pose severe health hazards for consumers. Agricultural produce may be contaminated by animal droppings, farmworkers' hands and irrigation water, etc. Fresh produce-related outbreaks can be controlled my managing agricultural and post-harvest practices [47]. The colonization of agronomically important plants by opportunistic human pathogens has been reported. For instance, the presence of B. cereus, $P$. aeruginosa and S. saprophyticus has been reported within the root system of plants with several plant growth-promoting attributes including IAA [48-50]. It has been reported that pathogenic species to animals and humans are mainly transmitted through the food chain. Therefore, pathogenic bacteria can contaminate plant surfaces and actively interact and colonize them as an alternate host [10]. Moreover, in previous studies, these bacteria have also been shown to exhibit beneficial plant attributes including IAA [51]. Bacterial genera such as Burkholderia, Enterobacter, Stenotrophomonas, Pseudomonas, Bacillus and Staphylococcus are associated with plants and affect their hosts in beneficial ways [11]. They have also been reported to stimulate plant growth by the production of IAA, biofilm formation or phosphate solubilization $[20,46]$. Thus, bacterial strains showing beneficial traits may be used as biofertilizers to minimize the use or cost of inorganic fertilizers for crop production. Phosphorus $(\mathrm{P})$ is the second most important macronutrient (after nitrogen) required for plant growth and development. Inadequate $P$ availability may cause stunted plant growth, and an abnormal dark green leaf color with reddish to purple tips or margins. Rhizobacteria can secrete organic acids or phosphatases in soils to convert 
insoluble P into soluble ions that can be utilized by plants [52]. In the current study, Pan. dispersa BPc-3 and B. subtilis Lb-61 were strongly positive for HCN (Table S2). Production of HCN by bacteria may play a very critical role in the suppression of phytopathogens [49].

\section{Conclusions}

In conclusion, fresh raw vegetables were colonized by a variety of bacterial genera. Although no obligate human pathogens were detected, the presence of a few members of potential human pathogens makes the biosafety of these vegetable questionable. They can cause harmful health effects to consumers. It may be difficult to completely remove these bacteria from food but certain precautionary measures particularly in agricultural practices, harvesting and processing can decrease the number of potential risk factors in these fresh produce products. Nevertheless, due to the close proximity with the plant surfaces, these microbes also harbor beneficial plant growth-promoting traits such as auxin production, mineral solubilization and biofilm formation. Overall, this study reported the association of several bacterial genera from the surfaces of agronomically important raw vegetables. The methodological strategies used in this study will help to investigate or screen general or specific bacterial diversity associated with other raw vegetables. In future, this study can be further extended to identify the indigenous bacterial communities associated with fresh agricultural produce growing in different geographical locations.

Supplementary Materials: The following are available online at http:/ / www.mdpi.com/2223-7747/8/4/91/s1, Figure S1: Purified bacterial colonies on LB agar, Figure S2: HCN production by different purified bacterial isolates, Figure S3: Phosphate solubilization by A. caloaceticus Eb-8 on Pikovskaya medium, Table S1: Auxin production and biofilm formation by plant associated bacteria, Table S2: Phosphate solubilization and HCN production by different bacterial isolates.

Author Contributions: B.A. developed the experimental layout, managed lab experiments, performed statistical analysis and wrote this manuscript.

Funding: This research received no external funding.

Acknowledgments: University of the Punjab, Lahore, Pakistan, is acknowledged for providing financial support for this research work.

Conflicts of Interest: The authors declare no conflict of interest.

\section{References}

1. Pem, D.; Jeewon, R. Fruit and vegetable intake: Benefits and progress of nutrition education interventions-Narrative review article. Iran. J. Public Health 2015, 44, 1309-1321. [PubMed]

2. Sivapalasingam, S.; Friedman, C.R.; Cohen, L.; Tauxe, R.V. Fresh produce: A growing cause of outbreaks of foodborne illness in the United States, 1973 through 1997. J. Food Prot. 2004, 67, 2342-2353. [CrossRef] [PubMed]

3. Heaton, J.C.; Jones, K. Microbial contamination of fruit and vegetables and the behavior of enteropathogens in the phyllosphere: A review. J. Appl. Microbiol. 2008, 102, 613-626. [CrossRef] [PubMed]

4. McMahon, M.A.S.; Wilson, I.G. The occurrence of enteric pathogens and Aeromonas species in organic vegetables. Int. J. Food Microbiol. 2001, 70, 155-162. [CrossRef]

5. Johannessen, G.S.; Loncarevic, S.; Kruse, H. Bacteriological analysis of fresh produce in Norway. Int. J. Food Microbiol. 2002, 77, 199-204. [CrossRef]

6. Salleh, N.A.; Rusul, G.; Hassan, Z.; Reezal, A.; Isa, S.H.; Nishibuchi, M.; Radu, S. Incidence of Salmonella spp. in raw vegetables in Selangor, Malaysia. Food Control 2003, 14, 475-479. [CrossRef]

7. Johnston, L.M.; Jaykus, L.A.; Moll, D.; Martinez, M.C.; Anciso, J.; Mora, B.; Moe, C.L. A field study on the microbiological quality of fresh produce. J. Food Prot. 2005, 68, 1840-1847. [CrossRef] [PubMed]

8. Mukherjee, A.; Speh, D.; Dyck, E.; Diez-Gonzalez, F. Preharvest evaluation of coliforms, Escherichia coli, Salmonella and Escherichia coli O157:H7 in organic and conventional produce grown by Minnesota farmers. J. Food Prot. 2004, 67, 894-900. [CrossRef] [PubMed]

9. Mukherjee, A.; Speh, D.; Jones, A.T.; Buesing, K.M.; Diez-Gonzalez, F. Longitudinal microbiological survey of fresh produce grown by farmers in the upper midwest. J. Food Prot. 2006, 69, 1928-1936. [CrossRef] 
10. Holden, N.; Pritchard, L.; Toth, I. Colonization outwith the colon: Plants as an alternative environmental reservoir for human pathogenic enterobacteria. FEMS Microbiol. Rev. 2009, 33, 689-703. [CrossRef] [PubMed]

11. Suckstorff, I.; Berg, G. Evidence for dose-dependent effects on plant growth by Stenotrophomonas strains from different origins. J. Appl. Microbiol. 2003, 95, 656-663. [CrossRef] [PubMed]

12. Bernier, S.P.; Silo-Suh, L.; Woods, D.E.; Ohman, D.E.; Sokol, P.A. Comparative analysis of plant and animal models for characterization of Burkholderia cepacia virulence. Infect. Immun. 2003, 71, 5306-5313. [CrossRef] [PubMed]

13. Teplitski, M.; Warriner, K.; Bartz, J.; Schneider, K.R. Untangling metabolic and communication networks: Interactions of enterics with phytobacteria and their implications in produce safety. Trends Microbiol. 2011, 19, 121-127. [CrossRef] [PubMed]

14. Mendes, R.; Garbeva, P.; Raajimakers, J.M. The rhizosphere microbiome: Significance of plant beneficial, plant pathogenic, and human pathogenic microorganisms. FEMS Microbiol. Rev. 2013, 37, 634-663. [CrossRef] [PubMed]

15. Lugtenberg, B.; Kamilova, F. Plant-growth-promoting rhizobacteria. Annu. Rev. Microbiol. 2009, 63, 541-556. [CrossRef] [PubMed]

16. Raaijmakers, J.M.; Paulitz, T.C.; Steinberg, C.; Alabouvette, C.; Moënne-Loccoz, Y. The rhizosphere: A playground and battlefield for soilborne pathogens and beneficial microorganisms. Plant Soil. 2009, 321, 341-361. [CrossRef]

17. Ahmed, A.; Hasnain, S. Auxin-producing Bacillus sp.: Auxin quantification and effect on the growth of Solanum tuberosum. Pure Appl. Chem. 2010, 82, 313-319. [CrossRef]

18. Lwin, K.M.; Myint, M.M.; Tar, T.; Aung, W.Z.M. Isolation of plant hormone (indole-3-acetic acid-IAA) producing rhizobacteria and study on their effects on maize seedling. Eng. J. 2012, 16, 137-144. [CrossRef]

19. Talboys, P.J.; Owen, D.W.; Healey, J.R.; Withers, P.J.; Jones, D.L. Auxin secretion by Bacillus amyloliquefaciens FZB42 both stimulates root exudation and limits phosphorus uptake in Triticum aestivum. BMC Plant Biol. 2014, 14, 51. [CrossRef]

20. Akhtar, S.; Ali, B. Evaluation of rhizobacteria as non-rhizobial inoculants for mung beans. Aust. J. Crop Sci. 2011, 5, 1723-1729.

21. Cappuccino, J.G.; Sherman, N. Microbiology: A Laboratory Manual; Pearson Education: Singapore, 2002.

22. Park, S.-H.; Ryu, S.; Kang, D.-H. Development of an improved selective and differential medium for isolatin of Salmonella spp. J. Clin. Microbiol. 2012, 50, 3222-3226. [CrossRef] [PubMed]

23. Johnson, J.L. Similarity analysis of rRNA. In Methods for General and Molecular Bacteriology; Gerhanrdt, P., Murray, R.G.E., Wood, W.A., Krieg, N.R., Eds.; American Society for Microbiology: Washington, DC, USA, 1994; pp. 625-700.

24. Tamura, K.; Stecher, G.; Peterson, D.; Filipski, A.; Kumar, S. MEGA6: Molecular Evolutionary Genetics Analysis version 6.0. Mol. Biol. Evol. 2013, 30, 2725-2729. [CrossRef] [PubMed]

25. Saitou, N.; Nei, M. The neighbor-joining method: A new method for reconstructing phylogenetic trees. Mol. Biol. Evol. 1987, 4, 406-425. [PubMed]

26. Bauer, A.W.; Kirby, W.M.; Sherris, J.C.; Turck, M. Antibiotic susceptibility testing by a standardized single disk method. Am. J. Clin. Pathol. 1966, 45, 493-496. [CrossRef] [PubMed]

27. Tang, W.Y.; Borner, J. Enzymes involved in synthesis and breakdown of indoleacetic acid. In Modern Methods of Plant Analysis; Paech, K., Tracey, M.V., Eds.; Springer: Heidelberg, Germany, 1979; Volume 7, pp. 238-241.

28. Pikovskaya, R.I. Mobilization of phosphorus in soil in connection with vital activity of some microbial species. Microbiologiya 1948, 17, 362-370.

29. Ahmad, F.; Ahmad, I.; Khan, M.S. Screening of free-living rhizospheric bacteria for their multiple plant growth promoting activities. Microbiol. Res. 2008, 163, 173-181. [CrossRef] [PubMed]

30. Christensen, G.D.; Simpson, W.A.; Younger, J.J.; Baddour, L.M.; Barrett, F.F.; Melton, D.M.; Beachey, E.H. Adherence of coagulase negative staphylococci to plastic tissue culture plates: A quantitive model for the adherence of staphylococci to medical devices. J. Clin. Microbiol. 1985, 22, 996-1006.

31. Berger, C.N.; Sodha, S.V.; Shaw, R.K.; Pink, D.; Hand, P.; Frankel, G. Fresh fruits and vegetables as vehicles for the transmission of human pathogens. Environ. Microbiol. 2010, 12, 2385-2397. [CrossRef]

32. Critzer, F.J.; Doyle, M.P. Microbial ecology of foodborne pathogens associated with produce. Curr. Opin. Biotechnol. 2010, 21, 125-130. [CrossRef] 
33. Schikora, A.; Gracia, A.V.; Hirt, H. Plants as alternative hosts for Salmonella. Trends Plant Sci. 2012, 17, 245-249. [CrossRef] [PubMed]

34. Arnesen, S.L.P.; Fagerlund, A.; Granum, P.E. From soil to gut: Bacillus cereus and its food poisoning toxins. FEMS Microbiol. Rev. 2008, 32, 579-606. [CrossRef] [PubMed]

35. Schoeni, J.L.; Wong, A.C. Bacillus cereus food poisoning and its toxins. J. Food Prot. 2005, 68, 636-648. [CrossRef] [PubMed]

36. Falomir, M.P.; Rico, H.; Gozalbo, D. Enterobacter and Klebsiella species isolated from fresh vegetables marketed in Valencia (Spain) and their clinically relevant resistances to chemotherapeutic agents. Foodborne Pathog. Dis. 2013, 10, 1002-1007. [CrossRef] [PubMed]

37. Argudin, M.A.; Mendoza, M.C.; Rodicio, M.R. Food poisoning and Staphylococcus aureus enterotoxins. Toxins 2010, 2, 1751-1773. [CrossRef] [PubMed]

38. Olsen, S.J.; MacKinon, L.C.; Goulding, J.S.; Slutsker, L. Surveillance for foodborne disease outbreaks-United States, 1993-1997. MMWR 2000, 49, 1-62. [PubMed]

39. Greene, S.K.; Daly, E.R.; Talbot, E.A.; Demma, L.J.; Holzbauer, S.; Patel, N.J.; Hill, T.A.; Walderhaug, M.O.; Hoekstra, R.M.; Lynch, M.F.; et al. Recurrent multistate outbreak of Salmonella Newport associated with tomatoes from contaminated fields, 2005. Epidemiol. Infect. 2008, 136, 157-165. [CrossRef]

40. Soon, J.M.; Manning, L.; Davies, W.P.; Baines, R. Fresh produce-associated outbreaks: A call for HACCP on farms? Br. Food J. 2010, 114, 553-597. [CrossRef]

41. Gumiere, T.; Ribeiro, C.M.; Vasconcellos, R.L.; Cardoso, E.J. Indole-3-acetic acid producing root-associated bacteria on growth of Brazil pine (Araucaria angustifolia) and slash pine (Pinus elliottii). Antonie Van Leeuwenhoek 2014, 105, 663-669. [CrossRef]

42. Fierro-Coronado, R.F.; Quiroz-Figueroa, F.R.; García-Pérez, L.M.; Ramírez-Chávez, E.; Molina-Torres, J.; Maldonado-Mendoza, I.E. IAA-producing rhizobacteria from chickpea (Cicer arientinum L.) induce changes in root architecture and increase root biomass. Can. J. Microbiol. 2014, 60, 639-648. [CrossRef]

43. Raheem, A.; Ali, B. Halotolerant rhizobacteria: Beneficial plant metabolites and growth enhancement of Triticum aestivum L. in salt amended soils. Arch. Agron. Soil Sci. 2015, 61, 1691-1705. [CrossRef]

44. Zaidi, A.; Ahmad, E.; Khan, M.S.; Saif, S.; Rizvi, A. Role of plant growth promoting rhizobacteria in sustainable production of vegetables: Current perspective. Sci. Hortic. 2015, 193, 231-239. [CrossRef]

45. Ali, B.; Sabri, A.N.; Hasnain, S. Rhizobacterial potential to alter auxin content and growth of Vigna radiata (L.). World J. Microniol. Biotechnol. 2010, 26, 1379-1384. [CrossRef]

46. Ramey, B.E.; Koutsoudis, M.; Bodman, S.B.; Fuqua, C. Biofilm formation in plant-microbe associations. Curr. Opin. Microbiol. 2004, 7, 602-609. [CrossRef] [PubMed]

47. Bartz, F.E.; Lickness, J.S.; Heredia, N.; De Aceituno, A.F.; Newman, K.L.; Hodge, D.W.; Jaykus, L.-A.; García, S.; Leon, J.S. Contamination of fresh produce by microbial indicators on farm and in packing facilities: Elucidation of environmental routes. Appl. Environ. Microbiol. 2017, 83, e02984-16. [CrossRef] [PubMed]

48. Aslam, F.; Ali, B. Halotolerant bacterial diversity associated with Suaeda fruticosa (L.) Forssk. improved growth of Maize under salinity stress. Agronomy 2018, 8, 131. [CrossRef]

49. Anwar, S.; Ali, B.; Sajid, I. Screening of rhizospheric actinomycetes for various in-vitro and in-vivo plant growth promoting (PGP) traits and for agroactive compounds. Front. Microbiol. 2016, 7, 1334. [CrossRef]

50. Egamberdieva, D.; Kamilova, F.; Validov, S.; Gafurova, L.; Kucharova, Z.; Lugtenberg, B. High incidence of plant growth-stimulating bacteria associated with the rhizosphere of wheat grown on salinated soil in Uzbekistan. Environ. Microbiol. 2008, 10, 1-9. [CrossRef] [PubMed]

51. Noreen, S.; Ali, B.; Hasnain, S. Growth promotion of Vigna mungo (L.) by Pseudomonas spp. exhibiting auxin production and ACC-deaminase activity. Ann. Microbiol. 2012, 62, 411-417. [CrossRef]

52. Sadiq, A.; Ali, B. Growth and yield enhancement of Triticum aestivum L. by rhizobacteria isolated from agronomic plants. Aust. J. Crop Sci. 2013, 7, 1544-1550.

(C) 2019 by the author. Licensee MDPI, Basel, Switzerland. This article is an open access article distributed under the terms and conditions of the Creative Commons Attribution (CC BY) license (http:/ / creativecommons.org/licenses/by/4.0/). 